\title{
Closed Geodesics and Billiards on Quadrics related to elliptic KdV solutions *
}

\author{
Simonetta Abenda \\ Dipartimento di Matematica e CIRAM \\ Università degli Studi di Bologna, Italy \\ abenda@ciram.unibo.it \\ and \\ Yuri Fedorov \\ Department of Mathematics and Mechanics \\ Moscow Lomonosov University, Moscow, 119 899, Russia \\ fedorov@mech.math.msu.su \\ and \\ Departament de Matematica I \\ Universitat Politecnica de Catalunya, Spain \\ Yuri.Fedorov@upc.es
}

November 20, 2018

\begin{abstract}
We consider algebraic geometrical properties of the integrable billiard on a quadric $Q$ with elastic impacts along another quadric confocal to $Q$. These properties are in sharp contrast with those of the ellipsoidal Birkhoff billiards in $\mathbb{R}^{n}$. Namely, generic complex invariant manifolds are not Abelian varieties, and the billiard map is no more algebraic. A Poncelet-like theorem for such system is known. We give explicit sufficient conditions both for closed geodesics and periodic billiard orbits on $Q$ and discuss their relation with the elliptic $\mathrm{KdV}$ solutions and elliptic Calogero system.
\end{abstract}

\section{Introduction}

One of the best known discrete integrable systems is the billiard inside an $n$ dimensional ellipsoid (more generally, a quadric)

$$
\begin{gathered}
Q=\left\{\frac{X_{1}^{2}}{a_{1}}+\cdots+\frac{X_{n+1}^{2}}{a_{n+1}}=1\right\} \in \mathbb{R}^{n+1}, \quad \mathbb{R}^{n}=\left(X_{1}, X_{2}, \ldots, X_{n+1}\right) \\
0<a_{1}<\cdots<a_{n}<a_{n+1}
\end{gathered}
$$

\footnotetext{
*AMS Subject Classification 37J60, 37J35, 70H45
} 
with elastic refections on $Q[5]$. The billiard inside $Q$ (Birkhoff case) inherits the remarkable property of geodesics on $Q$ given by the Chasles theorem: the trajectories or their continuations before and after impacts are tangent to the same set of $n$ quadrics that are confocal to $Q$ (see, e.g., [29]). The parameters of these quadrics deliver $n$ independent and commuting first integrals of the discrete system.

Veselov [36] described the Birkhoff billiard map in terms of discrete Lagrangian formalism and showed that its complex invariant manifolds are open subsets of coverings of Jacobians of hyperelliptic curves. Moreover, the restriction of the map to such manifolds is represented by shifts by a constant vector, which gives rise to an addition law on Abelian varieties.

Explicit theta function solutions for this billiard were obtained in 36 by applying spectral theory of difference operators, and later in [19] by finding a degeneration of theta function solutions for geodesics on an ellipsoid of a higher dimension.

The periodicity problem for the Birkhoff billiard map is closely related to a generalization of the following geometrical problem: given two smooth conics in the projective plane, $C$ and $D$, to construct a closed polygon inscribed in $C$ and circumscribed about $D$. The full Poncelet theorem states that, if a closed polygon exists for given $C$ and $D$, then every point of $C$ is a vertex of a closed polygon and all such polygons have the same number of sides [22].

Cayley theorem gives an explicit condition to find such a polygon. Namely, the iterative process of constructing the polygonal line is equivalent to shifts by a constant vector $\mathbf{v}$ in the Jacobian of an elliptic curve, $\mathcal{E}$. Then the polygonal line is closed after $n$ steps if and only if $\mathbf{v}$ is neutral on $\mathcal{E}(9,23])$.

The relation of the Poncelet problem to periodic billiard orbits inside $Q$ is evident, and such orbits are described by generalizations of the theorems of Poncelet and of Cayley in [12, 14, 15, 31].

On the other hand, one can consider geodesic motion on the ellipsoid $Q$ combined with elastic reflections along its intersections with the confocal quadric

$$
Q_{d}=\left\{\frac{X_{1}^{2}}{a_{1}-d}+\cdots+\frac{X_{n+1}^{2}}{a_{n+1}-d}=1\right\},
$$

$d$ being an arbitrary fixed parameter. Thus one obtains the billiard on the ellipsoid described by the map $\mathcal{B}:(x, v) \mapsto(\tilde{x}, \tilde{v})$, where $x, v \in \mathbb{R}^{n+1}$ are respectively the coordinates of an impact point on $Q \cap Q_{d}$ and the outgoing velocity at this point, whereas $\tilde{x}, \tilde{v}$ denote the same objects at the next impact point.

As shown in 13, this billiard map is also integrable and the trajectories between impacts (arches of geodesic) or their continuations are tangent to the same set of $n-1$ quadrics (caustics) in $\mathbb{R}^{n+1}$ that are confocal to $Q$ and $Q_{d}$. For $n=2$, algebraic geometrical features of this problem have already been discussed in [37.

Next, following 13, if a billiard trajectory of $Q$ is closed, then all the trajectories sharing the same caustics are closed as well and have the same period and length. Thus one arrives at a generalization of the celebrated Poncelet theorem on a quadratic surface. 
Contents of the paper. In this paper paper we study algebraic geometrical properties and periodic orbits of the billiard on the ellipsoid $Q$ with impacts along its intersection with a confocal quadric $Q_{d}$.

All our statements in the complex setting also hold in the case of a general quadric $Q$. On the other hand, in the description of real closed geodesics and periodic billiard orbits we always assume $Q$ to be an ellipsoid, since, for a generic real quadric $Q$, the billiard problem becomes meaningful only after a compactification.

We show that the algebraic-geometrical properties of the billiards on $Q$ with impacts along $Q_{d}$ are in sharp contrast with those of the Birkhoff case. Namely, the generic complex invariant manifolds are not Abelian varieties, but open subsets of theta-divisors of $n$-dimensional hyperelliptic Jacobians.

Next, although the billiard map $\mathcal{B}$ is integrable and all of its first integrals are algebraic, in the complex coordinates $(x, v)$ it is infinitely many valued, whereas in the real domain the new values $(\tilde{x}, \tilde{v})$ are determined by solving a system of transcendental equations which involve the inversion of hyperelliptic integrals.

To our knowledge, such billiards actually provide a first nontrivial example of a discrete integrable system which cannot be described in terms of an algebraic addition law on Abelian varieties.

Such systems can be regarded as discrete analogs of hyperelliptically separable systems, a class of finite-dimensional integrable differential equations whose generic complex solutions have movable algebraic branch points and are single-valued on an infinitely sheeted ramified covering of the complex plane $t$ (see, e.g., 38, 1, 2, 18]).

In Section 2 we briefly recall an algebraic geometrical description and thetafunction solution of the Jacobi problem on geodesics on a quadric $Q$, then describe the main properties of the billiard on $Q$.

In Section 3, we consider the periodicity problem for the billiard map $\mathcal{B}$ and we show that, in general, such conditions cannot be formulated in an algebraic form.

This however becomes possible for special initial conditions for which the corresponding hyperelliptic curve $\Gamma$ is a covering of an elliptic curve $\mathcal{E}$ and one of the holomorphic differential on $\Gamma$ reduces to that on $\mathcal{E}$. Under these conditions, the corresponding geodesics on $Q$ have a finite number of intersections with the boundary $Q \cap Q_{d}$ and therefore are closed.

In Section 4, we use known properties of the periodic KdV solutions and of the solutions to the elliptic Calogero system [17, 10, 4, 26, 32, 33, 20, 35] to describe the curves $\Gamma$ and the related closed geodesics on $Q$. As a result we show that to each elliptic $N$-soliton KdV solution or solution of the $N$-body elliptic Calogero systems satisfying the locus condition there corresponds a family of closed geodesics on a quadric $Q$.

We consider in detail the case of the $3: 1$ coverings $\Gamma \mapsto \mathcal{E}$ and obtain explicit sufficient conditions for a real geodesic on a 2-dimensional ellipsoid $Q$ to be closed.

Finally, in Section 5 we derive sufficient conditions for the periodicity of the billiard map on $Q$ by describing admissible boundary parameters $d$ of $Q_{d}$. To our knowledge, this is the first time a sufficient condition of periodicity for such billiard map is given. 
In the conclusion we outline possible extensions of this approach to periodic billiard orbits on a multi-dimensional ellipsoid $Q$.

\section{Geodesics and Billiards on a Quadric}

We start with the celebrated Jacobi problem of the geodesic motion on an $n$ dimensional ellipsoid $Q$. The problem is well known to be integrable and to be linearized on a covering of the Jacobian of a genus $n$ hyperelliptic curve. Namely, let $l$ be the natural parameter of the geodesic and $\lambda_{1}, \ldots, \lambda_{n}$ be the ellipsoidal coordinates on $Q$ defined by the formulas

$$
X_{i}=\sqrt{\frac{\left(a_{i}-\lambda_{1}\right) \cdots\left(a_{i}-\lambda_{n}\right)}{\prod_{j \neq i}\left(a_{i}-a_{j}\right)}}, \quad i=1, \ldots, n+1 .
$$

Then, denoting $\dot{\lambda}_{k}=d \lambda_{k} / d l$ the corresponding velocities, the total energy $\frac{1}{2}(\dot{X}, \dot{X})$ takes the Stäckel form

$$
H=2 \sum_{k=1}^{n} \frac{1}{\Psi\left(\lambda_{k}\right)} \prod_{j \neq k}^{n}\left(\lambda_{k}-\lambda_{j}\right) \dot{\lambda}_{k}^{2}, \quad \Psi(\lambda)=\left(\lambda-a_{1}\right) \cdots\left(\lambda-a_{n+1}\right) .
$$

According to the Stäckel theorem, the system is Liouville integrable and, after reparametrization

$$
d l=\lambda_{1} \cdots \lambda_{n} d s,
$$

the evolution of $\lambda_{i}$ is described by quadratures

$$
\begin{gathered}
\frac{\lambda_{1}^{k-1} d \lambda_{1}}{2 \sqrt{-\Psi\left(\lambda_{1}\right) R\left(\lambda_{1}\right)}}+\cdots+\frac{\lambda_{n}^{k-1} d \lambda_{n}}{2 \sqrt{-\Psi\left(\lambda_{n}\right) R\left(\lambda_{n}\right)}}=\left\{\begin{aligned}
d s & \text { for } k=1, \\
0 & \text { for } k=2, \ldots, n,
\end{aligned}\right. \\
R(\lambda)=\lambda\left(\lambda-c_{1}\right) \cdots\left(\lambda-c_{n-1}\right),
\end{gathered}
$$

$c_{k}$ being constants of motion. Then the components of the velocity have the form

$$
V_{i}=\frac{d X_{i}}{d l}=\frac{\sqrt{a_{i}\left(a_{i}-\lambda_{i}\right) \cdots\left(a_{i}-\lambda_{n}\right)}}{\sqrt{\prod_{j \neq i}\left(a_{i}-a_{j}\right)}} \sum_{k=1}^{n} \frac{1}{\prod_{j \neq k}\left(\lambda_{k}-\lambda_{j}\right)} \frac{\sqrt{-\Psi\left(\lambda_{k}\right) R\left(\lambda_{k}\right)}}{\lambda_{k}\left(a_{i}-\lambda_{k}\right)} .
$$

The quadratures involve $n$ independent holomorphic differentials on the genus $n$ hyperelliptic curve $\Gamma=\left\{\mu^{2}=-\Psi(\lambda) R(\lambda)\right\}$ and give rise to the Abel-Jacobi map of the $n$-th symmetric product $\Gamma^{(n)}$ to the Jacobian variety of $\Gamma$,

$$
\begin{gathered}
\int_{P_{0}}^{P_{1}} \omega_{k}+\cdots+\int_{P_{0}}^{P_{n}} \omega_{k}=u_{k}, \quad k=1, \ldots, n, \\
\omega_{k}=\frac{\lambda^{k-1} d \lambda}{2 \sqrt{-\Psi(\lambda) R(\lambda)}}, \quad P_{k}=\left(\lambda_{k}, \sqrt{-\Psi\left(\lambda_{k}\right) R\left(\lambda_{k}\right)}\right) \in \Gamma,
\end{gathered}
$$


where $u_{1}, \ldots, u_{n}$ are coordinates on the universal covering of $\operatorname{Jac}(\Gamma)$ and $P_{0}$ is a fixed basepoint, which we choose to be the infinity point, $\infty$, on $\Gamma$.

Since $u_{1}=s+$ const and $u_{2}, \ldots, u_{n}=$ const, the geodesic motion in the new parametrization is linearized on the Jacobian variety of $\Gamma$.

For the classical Jacobi problem $(n=2)$, its complete theta-functional solution was presented in 39], and, for arbitrary dimensions, in [25], whereas a complete classification of real geodesics was made in 3 .

Namely, let us fix a canonical homology basis on $\Gamma$ and let $\bar{\omega}_{1}, \ldots, \bar{\omega}_{n}$ be the conjugated basis of normalized holomorphic differentials,

$$
\bar{\omega}_{i}=\sum_{k=1}^{n} C_{i k} \omega_{k},
$$

$C_{i k}$ being normalization constants so that the $2 n \times n$ period matrix of $\Gamma$ has the form $\left(2 \pi_{\jmath} \mathbf{I}, B\right)$, where $B$ is the $n \times n$ Riemann matrix.

Let $\varphi=\left(\varphi_{1}, \ldots, \varphi_{n}\right)^{T}, \varphi_{i}=\sum_{s=1}^{n} C_{i s} u_{s}$ be the corresponding local coordinates on $\operatorname{Jac}(\Gamma)$ and

$$
\begin{gathered}
\theta\left[\begin{array}{l}
\alpha \\
\beta
\end{array}\right](\varphi \mid B)=\exp \{\langle B \alpha, \alpha\rangle / 2+\langle\varphi+2 \pi \jmath \beta, \alpha\rangle\} \theta(\varphi+2 \pi \jmath \beta+B \alpha), \\
\jmath=\sqrt{-1}
\end{gathered}
$$

be associated theta-function with characteristics $\alpha=\left(\alpha_{1}, \ldots, \alpha_{n}\right), \beta=\left(\beta_{1}, \ldots, \beta_{n}\right)$ and for $K, M \in \mathbb{Z}^{n}$,

$$
\begin{gathered}
\theta\left[\begin{array}{l}
\alpha \\
\beta
\end{array}\right](\varphi+2 \pi \jmath K+B M)=\exp (2 \pi \jmath \epsilon) \exp \{-(B M, M) / 2-(M, \varphi)\} \theta\left[\begin{array}{l}
\alpha \\
\beta
\end{array}\right](\varphi), \\
\epsilon=(\alpha, K)-(\beta, M) .
\end{gathered}
$$

Then the inversion of the map (2.5) applied to formulas (2.1) leads to the following parametrization of a generic geodesic

$$
\begin{gathered}
X_{i}(s)=\varkappa_{i} \frac{\theta\left[\Delta+\eta_{i}\right]\left(U_{1} s+\varphi_{0}\right)}{\theta[\Delta]\left(U_{1} s+\varphi_{0}\right)}, \quad i=1, \ldots, n+1, \\
U=2\left(C_{11}, \ldots, C_{n 1}\right) .
\end{gathered}
$$

Here $\Delta=\left(\delta^{\prime \prime}, \delta^{\prime}\right), \eta_{i}=\left(\eta_{i}^{\prime \prime}, \eta_{i}^{\prime}\right) \in \mathbb{R}^{2 n} / 2 \mathbb{R}^{2 n}$ are half-integer theta-characteristics such that modulo the period lattice of $\Gamma$ the following relations hold

$$
2 \pi \jmath \eta_{i}^{\prime \prime}+B \eta_{i}^{\prime}=\int_{\infty}^{\left(a_{i}, 0\right)}\left(\bar{\omega}_{1}, \ldots, \bar{\omega}_{n}\right)^{T}, \quad 2 \pi \jmath \delta^{\prime \prime}+B \delta^{\prime}=\mathcal{K}
$$

$\mathcal{K}$ being the vector of the Riemann constants, and $\kappa_{i}$ are constant factors depending on the moduli of $\Gamma$ only.

Remark 2.1. As follows from the quasiperiodic law (2.8), expressions (2.9) are meromorphic functions on a covering $\widetilde{\operatorname{Jac}}(\Gamma)$ of $\operatorname{Jac}(\Gamma)$ obtained by doubling some of its periods (see also [25]). 
The billiard on $Q$. We now describe the billiard map associated to the geodesic motion of a point on $Q$ with elastic bounces along the confocal quadric $Q_{d}$. By (2.1), when $\left(X_{1}, \ldots, X_{n+1}\right) \in Q \cap Q_{d}$, one of the points $P_{i}=\left(\lambda_{i}, \mu_{i}\right)$ on $\Gamma$ (without loss of generality we choose it to be $P_{n}$ ) coincides with one of the points $E_{d \pm}=$ $(d, \pm \sqrt{R(d)})$. Under the Abel-Jacobi map (2.5), the condition $P_{n}=E_{d \pm}$ defines two codimension one subvarieties in $\operatorname{Jac}(\Gamma)$, the theta-divisors

$$
\Theta_{d \pm}=\{\theta[\Delta](\varphi \mp C \mathfrak{q} / 2)\} \in \operatorname{Jac}(\Gamma),
$$

where $C=\left(C_{i k}\right)$ is the matrix introduced in (2.6) and $\mathfrak{q}$ is the vector

$$
\mathfrak{q}=\left(\mathfrak{q}_{1}, \ldots, \mathfrak{q}_{n}\right)^{T}=\int_{E_{d-}}^{E_{d+}}\left(\omega_{1}, \ldots, \omega_{n}\right)^{T} \in \mathbb{C}^{n}
$$

$\Theta_{d+}$ and $\Theta_{d-}$ have a common $(n-2)$-dimensional subvariety.

Hence, for fixed constants of motion, the coordinates $x$ of impact points on $Q \cap Q_{d}$ with velocities $v$ are described by degree $n-1$ divisors $\left\{P_{1}, \ldots, P_{n-1}\right\}$, that is, by a point $\varphi$ on $\Theta_{d-}$ or $\Theta_{d+}$.

An algebraic geometric description of the motion between impacts and at the elastic bounce along $Q \cap Q_{d}$ is given by the following proposition.

Proposition 2.1 The geodesic motion on $Q$ between subsequent impact points $x$ and $\tilde{x}$ on $Q \cap Q_{d}$ corresponds to the straight line uniform motion on $\operatorname{Jac}(\Gamma)$ between $\Theta_{d+}$ and $\Theta_{d-}$ along the $u_{1}$-direction. The point of intersection with $\Theta_{d-}$ gives the next coordinate $\tilde{x}$ and the ingoing velocity $v^{\prime}$. At the point $\tilde{x}$ the reflection $v^{\prime} \rightarrow \tilde{v}$ (from the ingoing to the outgoing velocity) results in jumping back from $\Theta_{d-}$ to $\Theta_{d+}$ by the shift vector $\mathfrak{q}$, which does not change $\tilde{x}$. Then the procedure iterates.

The process is sketched in Figure 1.

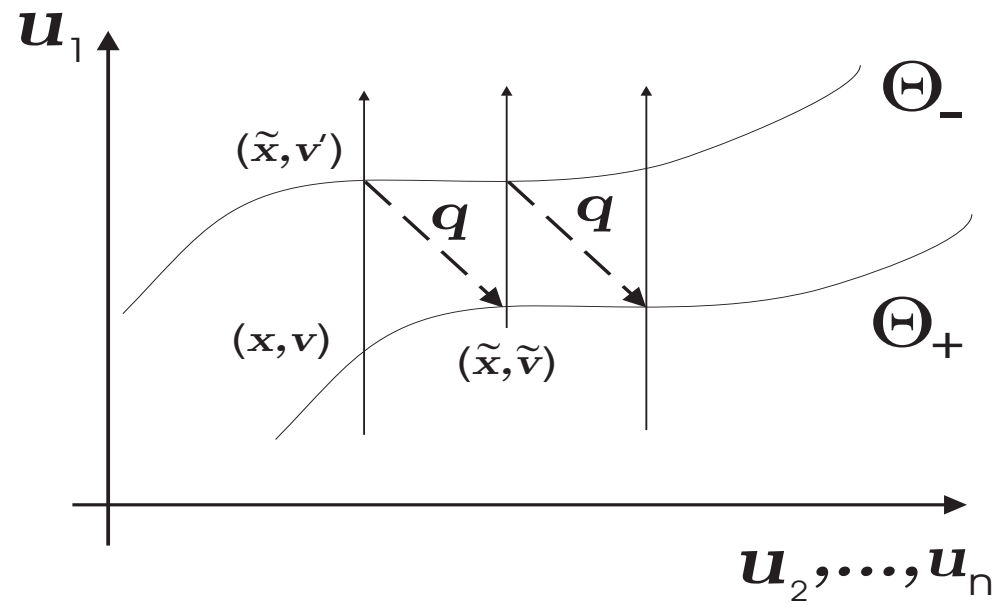

Figure 1

Proof of Proposition 2.1] First, we want to show that any subsequent impact points and the velocities $(x, v)$ and $\left(\tilde{x}, v^{\prime}\right)$ correspond to intersections of the flow with $\Theta_{d+}$ 
and $\Theta_{d-}$ respectively. For this purpose, we introduce the new time parameter $\Xi$ such that

$$
d \Xi=\frac{2 \sqrt{-\Psi(d) R(d)}}{\left(\lambda_{1}-d\right) \cdots\left(\lambda_{n}-d\right)} d s .
$$

Notice that in the real case the number $\sqrt{-\Psi(d) R(d)}$ is real and positive. Hence, as the point on $Q$ moves from $x$ to $\tilde{x}$, the parameter $\Xi$ changes monotonically from $-\infty$ to $\infty$. On the other hand, in view of the quadratures (2.3), one has

$$
d \Xi=\sum_{j=1}^{n} \frac{\sqrt{-\Psi(d) R(d)} d \lambda_{j}}{\left(\lambda_{i}-d\right) \sqrt{-\Psi\left(\lambda_{j}\right) R\left(\lambda_{j}\right)}}
$$

and, therefore,

$$
\Xi=\sum_{j=1}^{n} \int_{P_{0}}^{P_{j}} \frac{\sqrt{-\Psi(d) R(d)} d \lambda}{(\lambda-d) \sqrt{-\Psi\left(\lambda_{j}\right) R\left(\lambda_{j}\right)}}+\text { const. }
$$

The latter expression contains a meromorphic differential of the third kind on the curve $\Gamma$ having the pair of simple poles at $E_{ \pm}=(d, \pm \sqrt{-\Psi(d) R(d)})$ with residue \pm 1 respectively, hence the sum (2.12) is an Abelian transcendent of the third kind. According to the theory of theta-functions (see e.g., [1] or [7]), under the AbelJacobi map (2.5) the sum takes the form

$$
\Xi=\log \frac{\theta[\Delta](\varphi+C \mathfrak{q} / 2)}{\theta[\Delta](\varphi-C \mathfrak{q} / 2)}+\mathcal{C} s+\text { const }
$$

where $\mathcal{C}$ is a normalizing factor, $C=\left(C_{i k}\right)$ is defined in (2.6) and the vector $\mathfrak{q}$ is defined in (2.11). It follows that for $\Xi=-\infty$ and $\Xi=\infty$ the point $\varphi$ in $\operatorname{Jac}(\Gamma)$ belongs to $\Theta_{d+}$ and $\Theta_{d-}$ respectively, which proves the first statement of the theorem.

Next, we notice that for any $\varphi^{\prime} \in \Theta_{-}$and $\tilde{\varphi} \in \Theta_{+}$such that $\tilde{\varphi}=\varphi^{\prime}+C \mathfrak{q}$, the formula (2.9) gives one and the same Cartesian coordinates $X_{i}$. Indeed, under the mapping (2.5), $\tilde{\varphi}$ and $\varphi^{\prime}$ are represented by the degree $n$ divisors

$$
\left\{P_{1}=\left(\lambda_{1}, \mu_{1}\right), \ldots, P_{n-1}=\left(\lambda_{n-1}, \mu_{n-1}\right), E_{-}\right\}
$$

and, respectively, $\left\{P_{1}, \ldots, P_{n-1}, E_{+}\right\}$, which by (2.1), lead to the same values of $X_{i}$. On the other hand, expressions (2.4) imply that for $\tilde{\varphi}$ and $\varphi^{\prime}$ the velocities $V_{i}$ are different.

As a result, the intersection point $\varphi^{\prime} \in \Theta_{-}$corresponds to the pair $\left(\tilde{x}, v^{\prime}\right)$, whereas $\tilde{\varphi}=\varphi^{\prime}+C \mathfrak{q} \in \Theta_{+}$gives rise to $\tilde{x}$ and the outgoing velocity $\tilde{v}$.

It follows that the restriction of the billiard map $\mathcal{B}:(x, v) \rightarrow(\tilde{x}, \tilde{v})$ onto its invariant tori can be regarded as a transformation $\mathcal{B}_{c}: \Theta_{d+} \rightarrow \Theta_{d+}$. Then, as a corollary of Proposition 2.1, we obtain

Theorem 2.2 The map $\mathcal{B}_{c}$ is given by the shift of coordinates $u_{2}, \ldots, u_{n}$ by $\mathfrak{q}_{2}, \ldots, \mathfrak{q}_{n}$ respectively, whereas the first coordinate $u_{1}$ on $\operatorname{Jac}(\Gamma)$ is chosen such that the vector $\tilde{u}=\mathcal{B}_{c}(u)$ remains on the theta-divisor $\Theta_{d+}$.

Indeed, according to Proposition 2.1 (we recall that $\varphi=C u$ ), under the straight line flow on the Jacobian from $\Theta_{d+}$ to $\Theta_{d-}$, the coordinates $u_{2}, \ldots, u_{n}$ remain unchanged, and under the passage to $\Theta_{d+}$ they increase by $\mathfrak{q}_{2}, \ldots, \mathfrak{q}_{n}$. 
Remark 2.2. For $n=2$, the above algebraic geometrical property has already been described in [37]. The algebraic geometric description of the billiard on $Q$ stands in sharp contrast to the one of the Birkhoff billiard system - as well as to most of the known integrable maps. Indeed in the Birkhoff case, the billiard map is given by a shift by a constant vector on a complex invariant manifold which is an open subset of an Abelian variety. In our case, the shift takes place on a non-Abelian variety and, as a result, it is not fixed.

Branching of the map $\mathcal{B}_{c}$. The above properties of the map $\mathcal{B}$ cannot be used to derive an explicit expression for the billiard map $(x, v) \rightarrow(\tilde{x}, \tilde{v})$, since the complex map $\mathcal{B}_{c}: \Theta_{d+} \rightarrow \Theta_{d+}$ described by Theorem 2.2 is infinitely many-valued. Indeed, let $\widetilde{\Theta}_{d+}$ be the universal covering of $\Theta_{d+}$ in $\mathbb{C}^{n}=\left(u_{1}, \ldots, u_{n}\right)$ and let $\pi$ denote the natural projection $\widetilde{\Theta}_{d+} \rightarrow \mathbb{C}^{n-1}=\left(u_{2}, \ldots, u_{n}\right)$.

Theorem 2.3 (see also [1]). Suppose that the curve $\Gamma$ does not have nontrivial symmetries and its Jacobian does not possesses Abelian subvarieties, then

1). Under the projection $\pi, \widetilde{\Theta}_{d+}$ is an infinitely sheeted covering of $\mathbb{C}^{n-1}$ ramified along a codimension one subvariety of $\widetilde{\Theta}_{d+}$.

2). A complete preimage of any point in $\mathbb{C}^{n-1}$ consists of an infinite number of points on $\widetilde{\Theta}_{d+}$, which, in turn, represent an infinite number of points on $\Theta_{d+}$.

Proof. Let $\mathfrak{v}_{1}, \ldots, \mathfrak{v}_{2 n} \in \mathbb{C}^{n-1}=\left(u_{2}, \ldots, u_{n}\right)$ be the projections of $2 n$ independent period vectors of $\operatorname{Jac}(\Gamma)$. For any point $\mathcal{P} \in \Theta_{d+}$, the projections of points of its equivalence class on $\widetilde{\Theta}_{d+}$ have the form

$$
\left\{\pi(\mathcal{P})+\sum_{j=1}^{2 n} m_{j} \mathfrak{v}_{j} \mid m_{j} \in \mathbb{Z}\right\} .
$$

Let $\hat{z}$ be any fixed point on $\mathbb{C}^{n-1}$. Under the condition of the theorem, the projections $\mathfrak{v}_{j}$ are incommensurable and the integer coefficients $m_{j}$ may always be chosen in such a way that the above sum represents a point in any small and a priori defined neighborhood of $\hat{z}$. In this sense, any point on $\Theta_{+}$is projected into such a neighborhood of any point on $\mathbb{C}^{n-1}$. Hence, a complete preimage $\pi^{-1}$ of any point on $\mathbb{C}^{n-1}$ corresponds to an infinite number of points on the theta-divisor.

In the particular case $n=2$, when the theta-divisor or its translate coincides with the genus 2 curve $\Gamma$ itself, the above property is known in connection with the inversion of a single hyperelliptic integral

$$
\int_{\left(\lambda_{0}, w_{0}\right)}^{(\lambda, w)} \frac{d \lambda}{w}=u_{1}, \quad(\lambda, w) \in \Gamma .
$$

Namely, as was shown by Jacobi (see, e.g., 28]), the covering $\widetilde{\Gamma} \rightarrow \mathbb{C}=\left\{u_{1}\right\}$ has infinitely many branch points whose projections onto the complex plane $u_{1}$ form a dense set. 
Hence, given fixed coordinates $u_{2}, \ldots, u_{n}$ on $\operatorname{Jac}(\Gamma)$, there is an infinite number of $\tilde{u}_{1}$, which define the next impact point $\tilde{x} \in Q \cap Q_{d}$ via Proposition 2.1. In particular, this implies that the billiard map $\mathcal{B}:(x, v) \rightarrow(\tilde{x}, \tilde{v})$ cannot be algebraic.

The above phenomenon is also explained by the fact that a generic geodesic on $Q$ intersects any connected component of the boundary $Q \cap Q_{d}$ at infinitely many points. In the real domain, given $x \in Q \cap Q_{d}$, it is possible to determine $\tilde{x}$ by choosing the next intersection with the boundary, but in the complex setting such a choice cannot be made.

Remark 2.3. Due to the above properties, the billiard map $\mathcal{B}$ can be regarded as a discrete analog of the hyperelliptically separable systems studied, in particular, in 138, 1, 2, 18). Such systems, although being Liouville integrable, are not algebraic completely integrable: their generic complex solutions have movable algebraic branch points and are single-valued on an infinitely sheeted ramified covering of the complex plane $t$, so they possess the so called weak Painlevé property. The branching of the billiard map $\mathcal{B}$ can be viewed as a discrete version of such a property (see also 21]).

\section{Periodic Billiard Orbits on $Q \cap Q_{d}$}

We now concentrate on algebraic conditions for a billiard trajectory on the quadratic surface $Q$ to be periodic.

As mentioned in the Introduction, the Birkhoff billiard in the ellipsoid $Q \subset \mathbb{R}^{n+1}$ is described by translations on the Jacobians of genus $n$ hyperelliptic curves

$$
\Gamma=\left\{y^{2}=-R(x)\right\} \subset \mathbb{R}^{2}=(x, y), \quad R(x)=\left(x-b_{1}\right) \cdots\left(x-b_{2 n+1}\right) .
$$

Then the corresponding billiard trajectory is periodic if, for a certain integer $m$, the vector

$$
m \int_{E_{0-}}^{E_{0+}}\left(\omega_{1}, \ldots, \omega_{n}\right)^{T}, \quad E_{0 \pm}=(0, \pm \sqrt{-R(0)})
$$

is neutral in $\operatorname{Jac}(\Gamma)$. The periodicity conditions written explicitly in terms of the coefficients of the curve $\Gamma$ were obtained in [15] by proving the following theorem.

Theorem 3.1 ([15]) For a constant $d \in \mathbb{C}$ and involutive points $E_{d \pm}=(d, \pm \sqrt{R(d)}) \in$ $\Gamma$, the vector $m \int_{E_{d-}}^{E_{d+}} \omega(m>n)$ is neutral in $\operatorname{Jac}(\Gamma)$ if and only if

$$
\operatorname{rank}\left(\begin{array}{cc}
S_{m+1} & S_{m} \cdots S_{n} \\
S_{m+2} & S_{m+1} \cdots S_{n+1} \\
\vdots & \vdots \cdot \vdots \\
S_{2 m-1} & S_{2 m-2} \cdots S_{m+n}
\end{array}\right)<m-n
$$

where the entries $S_{j}$ are determined from the expansion

$$
\sqrt{\left(x-b_{1}\right) \cdots\left(x-b_{2 n+1}\right)}=S_{0}+S_{1}(x-d)+S_{2}(x-d)^{2}+\cdots .
$$


This theorem provides a multidimensional generalization of Cayley's algebraic condition for the existence of a polygon inscribed in a conic and circumscribed about another conic.

For the billiard on the quadric $Q$, the situation is quite different: generic complex invariant manifolds are no more Abelian varieties, but theta-divisors. As follows from Theorem 2.2. the billiard trajectory on $Q$ is periodic if for some integers $m, m_{1}, \ldots, m_{2 n}$ the following equality holds

$$
m\left(\mathfrak{q}_{2}, \ldots, \mathfrak{q}_{n}\right)^{T}=\sum_{j=1}^{2 n} m_{j} \mathfrak{v}_{j},
$$

Since the projections are generally incommensurable, the above condition can always be fulfilled, hence in the complex setting this criterion for closed billiard trajectories makes no sense.

Remark 3.1. On the other hand, it seems natural to try to replace the above condition with the following relation in the Jacobian:

$$
m \int_{E_{d-}}^{E_{d+}}\left(\omega_{1}, \ldots, \omega_{n}\right)^{T} \equiv 0 .
$$

That is, although the map $\mathcal{B}_{c}$ is not given by a shift by $\mathfrak{q}$ on $\operatorname{Jac}(\Gamma)$, it is required that after $N$ iterations it yields an identity map on $\operatorname{Jac}(\Gamma)$ and therefore, on the thetadivisor $\Theta_{d+}$ as well. This condition was proposed, amongst other results, in the recent paper [16], and explicit algebraic conditions on the coefficients of the curve $\Gamma$ were derived from it. However, since a generic geodesic $X(s) \subset Q$ intersects $Q \cap Q_{d}$ at infinitely many points, these conditions do not guarantee that the corresponding closed billiard trajectory consists of pieces of geodesics joining the nearest points of intersection with $Q \cap Q_{d}$.

Periodic billiard orbits related to closed geodesics. The projections $\mathfrak{v}_{1}, \ldots, \mathfrak{v}_{2 n}$ may become commensurable and the closeness condition in the complex domain may become meaningful if the hyperelliptic curve $\Gamma$ has certain symmetries and its Jacobian contains Abelian subvarieties, as described by the Weierstrass-Poincaré theory of reduction of Abelian varieties and functions (see e.g., [27] and, for modern exposition, [7, 6]).

In this paper we concentrate on the case when the genus $n$ hyperelliptic curve $\Gamma_{n}$ is an $N$-fold covering of an elliptic curve $\mathcal{E}$ and the "first" holomorphic differential $\omega_{1}=d \lambda / \mu$ reduces to a holomorphic differential on $\mathcal{E}$.

Under these conditions, the $u_{1}$-flow on $\operatorname{Jac}(\Gamma)$ intersects $\Theta_{d+}$ at a finite number of points. As a result, the corresponding geodesics on $Q$ also have a finite number of intersections with the boundary $Q \cap Q_{d}$ and therefore are closed.

According to the Poincare theorem, the existence of covering $\Gamma \mapsto \mathcal{E}$ implies certain conditions on the components of the Riemann matrix of the curve $\Gamma$ ( 7 , 6]). However, our objective below is to give sufficient conditions in terms of the coefficients of the polynomial $R(\lambda)$. 
Remark 3.2. We emphasize that not any covering $\Gamma \mapsto \mathcal{E}$ corresponds to closed geodesics. For example, in the simplest possible case $n=2, N=2$ studied by Jacobi and widely described in the literature (see, e.g. [27, 7]), the curve $\Gamma$ is birationally equivalent to the canonical curve

$$
w^{2}=z(z-1)(z-\alpha)(z-\beta)(z-\alpha \beta)
$$

$\alpha, \beta$ being arbitrary constant. It covers two elliptic curves

$$
\ell_{ \pm}=\left\{W_{ \pm}^{2}=Z_{ \pm}\left(1-Z_{ \pm}\right)\left(1-k_{ \pm} Z_{ \pm}\right)\right\}, \quad k_{ \pm}^{2}=-\frac{(\sqrt{\alpha} \mp \sqrt{\beta})^{2}}{(1-\alpha)(1-\beta)}
$$

and both of the holomorphic differentials $\omega_{1}, \omega_{2}$ on $\Gamma$ reduce to linear combinations of the holomorphic differentials on $\ell_{+}$and $\ell_{-}$.

In this case the inversion of the quadratures (2.3) and formulas (2.9) lead to solutions in terms of elliptic functions of $\ell_{ \pm}$, but, since their periods are generally incommensurable, the corresponding geodesics are quasi-periodic. Such geodesics were already studied in 8 .

\section{Elliptic KdV solutions, elliptic Calogero systems and closed geodesics on a quadric}

The most classical series of hyperelliptic tangential coverings $\mathcal{G}_{n} \rightarrow \mathcal{E}$ (see 34]) arises in the spectral theory of the Lamé potentials or as a subclass of spectral curves of the elliptic Calogero (EC) systems, which describe the motion of $N$ particles with coordinates $q_{1}, \ldots, q_{N}$ on the circle under the potential $\sum_{i<j}^{N} \wp\left(q_{i}-q_{j}\right)$, see [10]. Here $\wp(q)=\wp\left(q \mid \omega, \omega^{\prime}\right)$ denotes the Weierstrass elliptic function with half-periods $\omega, \omega^{\prime}$.

The EC systems are integrable and, as shown in [26, they possess a matrix $N \times N$ Lax pair with an elliptic spectral parameter $u \in \mathcal{E}=\mathbb{C} /\left\{2 \omega \mathbb{Z}+2 \omega^{\prime} \mathbb{Z}\right\}^{1}$,

$$
\frac{d}{d t} L(u)=[L(u), M(u)]
$$

The spectral curve $\mathcal{G}=|L(u)-\mu \mathbf{I}|=0$ (the so called Krichever curve) is an $N$-fold covering of the torus $\mathcal{E}$ and its genus does not exceed $N$.

The EC systems possess invariant subvarieties given by the $K d V$ locus condition

$$
\sum_{i \neq j}^{N} \wp^{\prime}\left(q_{i}-q_{j}\right)=0, \quad q_{i} \neq q_{j}, \quad j=1, \ldots, N .
$$

If $N$ equals a "triangular number", i.e., $N=n(n+1) / 2$ for some $n$, and the coordinates $q_{i}$ satisfy the KdV locus condition, then the following properties hold

\footnotetext{
${ }^{1}$ We do not give the explicit expressions for $L(u)$ here
} 
1). ([17, 4]) For a certain constant $C$, the function

$$
U(x, t)=2 \sum_{i=1}^{N} \wp\left(x-q_{i}(t)\right)+C
$$

is an elliptic solution of the $\mathrm{KdV}$ equation $U_{t}=6 U U_{x}-U_{x x x}$.

2). (32, 33] ) The Krichever spectral curve is hyperelliptic of genus $n$ : there exists a birational change of variables $(\wp(u), \mu) \rightarrow(z, w)$ such that the equation defining $\mathcal{G}$ takes the canonical form

$$
w^{2}=R_{2 n+1}(z) \equiv\left(z-z_{1}\right) \cdots\left(z-z_{2 n+1}\right)
$$

with some parameters $z_{k}$. Up to a constant factor, the last holomorphic differential $\omega_{n}=z^{n-1} d z / w$ on $\mathcal{G}$ reduces to the holomorphic differential $d \wp / \wp^{\prime}$ on $\mathcal{E}$.

3). (26]) Let $\psi_{1}, \ldots, \psi_{n-1}, \psi_{n}$ be the local coordinates on $\operatorname{Jac}(\mathcal{G})$ associated to the holomorphic differentials $\omega_{k}=z^{k-1} d z / w, k=1, \ldots, n$ on $\mathcal{G}$ and let $\varphi_{1}, \ldots, \varphi_{n}$ be the coordinates associated to the normalized holomorphic differentials. Let $\theta(\varphi)$ be the theta-function associated to $\mathcal{G}$ and $U \in \mathbb{C}^{n}$ be the tangent vector of the curve $\mathcal{G} \subset \operatorname{Jac}(\mathcal{G})$ at $\infty \in \mathcal{G}$, which in the coordinates $\psi_{k}$ has the form $U=(0, \ldots, 0,2)^{T}$. Then for suitable constant vectors $V, W \in \mathbb{C}^{n}$, the transcendental equation

$$
\theta[\Delta](U x+V t+W)=0, \quad q, t \in \mathbb{C},
$$

has exactly $N$ solutions $x=q_{1}(t), \ldots, x=q_{N}(t)$ on $\operatorname{Jac}(\mathcal{G})$, which provide the solution for the EC system.

4). (20]) One can choose a canonical homology basis on $\mathcal{G}$ and the basis of normalized holomorphic differentials such that the vectors $U, V$ in (4.4) take the form

$$
U=(1, \ldots, 0,0)^{T}, \quad V=\left(0, V_{1}, \ldots, V_{n-1}\right)^{T}
$$

and the period matrix of $\mathcal{G}$ becomes

$$
(2 \pi \jmath \mathbf{I} B), \quad B=\left(\begin{array}{ccccc}
\tau / N & 2 \pi \jmath / N & 0 & \cdots & 0 \\
2 \pi \jmath / N & & & & \\
0 & & & & \\
\vdots & & & \mathfrak{B} & \\
0 & & &
\end{array}\right),
$$

where $(2 \pi \jmath, \tau)$ is the normalized period matrix of the elliptic curve $\mathcal{E}$ and $\mathfrak{B}$ is a $(n-1) \times(n-1)$ Riemann matrix.

In view of normalization (4.5), the function $\theta[\Delta](U x+W)$ can be written as $\theta[\Delta](x, \mathbf{t}), \mathbf{t}=\left(t_{2}, \ldots, t_{n}\right)$. It is a one-dimensional theta-function of order $N$ 
with respect to $x$ and it admits the factorization

$$
\theta[\Delta](x, \mathbf{t})=\zeta(\mathbf{t}) \prod_{j=1}^{N} \theta_{11}\left(x-q_{j}(\mathbf{t})\right),
$$

where $\theta_{11}(x \mid \tau) \equiv \theta\left[\begin{array}{l}1 / 2 \\ 1 / 2\end{array}\right](x \mid \tau)$ is the one-dimensional Riemann thetafunction associated to $\mathcal{E}$ and the factor $\zeta(\mathbf{t})$ depends on $\mathbf{t}$ only.

Notice that item (3) provides an implicit solution for the EC system. Explicit expressions for certain symmetric functions of the coordinates $q_{i}$ were first given in 20].

According to the Poincaré reducibility theorem (see e.g., [7]), apart from the curve $\mathcal{E}$, the Jacobian of $\mathcal{G}$ contains an $(n-1)$-dimensional Abelian subvariety $\mathcal{A}_{n-1}$ and is isogenous to the direct product $\mathcal{E} \times \mathcal{A}_{n-1}$.

Notice that for $n=2$, explicit algebraic expressions of the covers and coefficients of hyperelliptic curves are known for $N \leq 8$ (see [35]).

Remark 4.1. Property 3) also implies that the $x$-flow (or $\psi_{n}$-flow) on $\operatorname{Jac}(\mathcal{G})$, which is tangent to $\mathcal{G} \subset \mathrm{J} a c(\mathcal{G})$ at its infinity point $\infty$, intersects any translate of the theta-divisor $\Theta \subset \operatorname{Jac}(\mathcal{G})$ precisely at $n(n+1) / 2$ points (possibly with multiplicity).

This property is similar to what we require for the linearization of the geodesic flow on the $n$-dimensional quadric $Q$. However, as seen from the quadratures (2.3), the linearized flow on $\operatorname{Jac}(\Gamma)$ has a different direction: it is tangent to the embedded hyperelliptic curve $\Gamma \subset \operatorname{Jac}(\Gamma)$ not at $\infty$, but at the Weierstrass point $\mathcal{O}=(0,0)$. Nevertheless, both behaviors are equivalent: if $(\beta, 0)$ is a finite Weierstrass point on $\mathcal{G}$, then by a birational transformation

$$
\lambda=\frac{\alpha}{(z-\beta)}, \quad \mu=\frac{w}{(z-\beta)^{n+1}}
$$

the points $\infty$ and $(\beta, 0)$ on $\mathcal{G}$ are sent to $\mathcal{O}$ and $\infty$ on $\Gamma$, respectively. Then, identifying the curves $\mathcal{G}$ and $\Gamma$ as well as their Jacobians, we find that the KdV flow $\left(\psi_{n}\right.$-flow) on $\operatorname{Jac}(\mathcal{G})$ is represented as $u_{1}$-flow on $\operatorname{Jac}(\Gamma)$ and vice versa.

Thus, the birational transformation (4.8) establishes a relation between the elliptic $N$-soliton KdV solutions (4.2) or the solutions of the $N$-body elliptic Calogero systems satisfying the locus condition and the geodesics on a quadric $Q$ that are linearized on $\operatorname{Jac}(\Gamma)$. Moreover, since the KdV solutions are periodic, all such geodesics are closed.

Clearly, for the geodesic and the quadric (ellipsoid) to be real, certain reality conditions on the coefficients of $\mathcal{G}$ must be satisfied.

In particular, assume that the roots $z_{1}, \ldots, z_{2 n+1}$ of $\mathcal{G}$ (see formula (4.3) have such values that the above transformation with certain $\alpha$ and $\beta=z_{2 n+1}$ sends them to real positive numbers $e_{1}, \ldots, e_{2 n}$ and the infinity. In this case one can always choose a partition

$$
\left\{e_{1}, \ldots, e_{2 n}\right\}=\left\{a_{1}, \ldots, a_{n+1}\right\} \cup\left\{c_{1}, \ldots, c_{n-1}\right\}
$$


such that the quadratures (2.3) describe the real closed geodesics on the ellipsoid $Q$ that are tangent to the confocal quadrics $Q\left(c_{1}\right), \ldots, Q\left(c_{n-1}\right)$.

Parametrization of the closed geodesics. Let the hyperelliptic curve $\Gamma$ be isomorphic to $\mathcal{G}$ via (4.8). Then, in view of property (4) of the EC solutions, we choose local coordinates $\varphi_{1}, \ldots, \varphi_{n}$ on $\operatorname{Jac}(\Gamma)$ such that its period matrix has the form (4.6) and the function $\theta[\Delta]\left(U_{1} s+\varphi_{0} \mid B\right)$ defined in (2.9), (2.10) can be written as $\theta[\Delta](s, \mathbf{t} \mid B), \mathbf{t}=\left(t_{2}, \ldots, t_{n}\right)^{T}=$ const. Here, as above, $s$ is the new parameter introduced in (2.2) and $\mathbf{t}$ plays the role of the constant phase vector numerating the geodesic.

Now let

$$
\left\{q_{1}(\mathbf{t}), \ldots, q_{N}(\mathbf{t})\right\} \quad \text { and } \quad\left\{p_{1 i}(\mathbf{t}), \ldots, p_{N i}(\mathbf{t})\right\}, \quad i=1, \ldots, n
$$

be solutions to equations

$$
\theta[\Delta](s, \mathbf{t})=0, \quad \text { respectively } \quad \theta\left[\Delta+\eta_{i}\right](s, \mathbf{t})=0, \quad s \in \mathbb{C} .
$$

Proposition 4.1 1). The Cartesian coordinates $X_{i}$ of the closed geodesics admit the following parametrization in terms of theta-functions of $\mathcal{E}$ :

$$
X_{i}(s \mid \mathbf{t})=\xi_{i}(\mathbf{t}) \exp (s / 2) \cdot \frac{\theta_{11}\left(s-p_{1 i}(\mathbf{t})\right) \cdots \theta_{11}\left(s-p_{N i}(\mathbf{t})\right)}{\theta_{11}\left(s-q_{1}(\mathbf{t})\right) \cdots \theta_{11}\left(s-q_{N}(\mathbf{t})\right)} .
$$

Here the factors $\xi_{i}(\mathbf{t})$ depend on the phases only, and, for any $\mathbf{t}$, modulo the periods $(2 \pi \jmath, \tau)$ of $\mathcal{E}$,

$$
p_{1 i}+\cdots+p_{N i} \equiv q_{1}+\cdots+q_{N}+\tau / 2 .
$$

2). Let the parameter $d \notin\left\{a_{1}, \ldots, a_{n+1}, c_{1}, \ldots, c_{n-1}\right\}$ be such that the real closed geodesics on $Q$ have a non-empty intersection with the quadric $Q_{d}$. Then the geodesics intersect each connected component $D_{d, j}$ of $Q \cap Q_{d}$ at least at 2 and at most at $n(n+1)$ distinct points.

As follows from (4.10), (4.11), as well as the quasi-periodic law of $\theta_{11}(s)$, when the argument $s$ changes by the period $2 \pi j$ of $\mathcal{E}$, the coordinates $X_{i}$ change sign, and they remain unchanged under the shift $s \rightarrow s+\tau$. Hence the corresponding closed geodesic is a 2-fold unramified covering of $\mathcal{E}$, i.e., it is an algebraic curve in $\mathbb{R}^{n+1}$.

Proof of Proposition 4.1. 1). Due to definition of generic theta-functions with characteristics (2.7), one has

$$
\theta\left[\Delta+\eta_{i}\right](\varphi)=\exp \left\{\left\langle\eta_{i}^{\prime}, B \eta_{i}^{\prime}\right\rangle+\left\langle\varphi+2 \pi \jmath\left(\eta_{i}^{\prime \prime}+\Delta_{i}^{\prime \prime}\right), \eta_{i}^{\prime}\right\rangle\right\} \theta[\Delta]\left(\varphi+2 \pi \jmath \eta_{i}^{\prime \prime}+B \eta_{i}^{\prime}\right)
$$

and therefore,

$$
\begin{aligned}
& \theta\left[\Delta+\eta_{i}\right](s, \mathbf{t})=\varkappa_{i}(\mathbf{t}) \exp \left(s\left(\eta_{i}\right)_{1}^{\prime}\right) \\
& \cdot \theta[\Delta]\left(s+\tau \frac{\left(\eta_{i}\right)_{1}^{\prime}}{N}+2 \pi \jmath\left[\frac{\left(\eta_{i}\right)_{2}^{\prime}}{N}+\left(\eta_{i}\right)_{1}^{\prime \prime}\right], \mathbf{t}+\mathfrak{t}\right)
\end{aligned}
$$


with certain factors $\varkappa_{i}(\mathbf{t})$ and vector $\mathfrak{t} \in \mathbb{C}^{n-1}$. One can show that, for this choice of the basis of cycles and of the normalized differentials on $\Gamma$, one always has $\left(\eta_{i}\right)_{1}^{\prime}=$ $1 / 2$. Next, in view of (4.9) and (4.7), the theta-function in the right hand side of (4.12) admits factorization in one-dimensional theta-functions $\theta_{11}\left(s-p_{k i} \mid \tau\right)$. Now applying this to the solution (2.9) for the Cartesian coordinates of a generic geodesic, one arrives at expressions (4.10).

Since the geodesics are closed, these expressions must be doubly periodic in $s$. Then, due to the quasiperiodic law for $\theta_{11}(s)$, relation (4.11) must hold.

2). As shown in Section 2, under the Abel-Jacobi map (2.5) the intersections of the geodesic with $Q \cap Q_{d}$ correspond to points on the two translates $\Theta_{d-}, \Theta_{d+}$ of the theta-divisor on $\operatorname{Jac}(\Gamma)$. Next, according to property 3 ) of the EC solutions, the $u_{1}$-flow on $\operatorname{Jac}(\Gamma)$ intersects any translate of $\Theta$ at $N=n(n+1) / 2$ (complex) points.

According to Remark 2.1., the geodesic flow is linearized on a covering $\widetilde{\operatorname{Jac}}(\Gamma)$ obtained by doubling of some of its periods. As a result, the $u_{1}$-flow on $\widetilde{\operatorname{Jac}}(\Gamma)$ has in total $4 N=2 n(n+1)$ intersections with the corresponding coverings of $\Theta_{d-} \cup \Theta_{d+}$, which gives rise to the same number of complex intersections of the geodesic with $Q \cap Q_{d}$.

For $d \neq a_{i}$, the real manifold $Q \cap Q_{d}$ consists of 2 connected components $D_{d, 1}, D_{d, 2}$. Then, due to symmetry, each of the components is intersected at most at $n(n+1)$ points.

The case $N=3, n=2$. In this simplest case the connected components $D_{1, d}, D_{2, d}$ are ovals which bound simply-connected domains $\mathcal{D}_{1, d}, \mathcal{D}_{2, d}$ on the 2-dimensional ellipsoid $Q$. Then one can define ingoing and outgoing intersections of the geodesic with $D_{i, d}$ (when the geodesic goes inside, respectively outside of $\mathcal{D}_{i, d}$ ). In view of Proposition 2.1] the former ones correspond to the intersections of the $u_{1}$-flow on $\operatorname{Jac}(\Gamma)$ with $\Theta_{d+}$, whereas the latter correspond to the intersections with the other translate $\Theta_{d-}$.

As follows from item (2) of Theorem 4.1 the closed geodesic has 3 ingoing and 3 outgoing complex intersections with each component $D_{i, d}$. If $d \rightarrow a_{i}$, both components are confluent to the ellipse $Q \cap\left\{X_{i}=0\right\}$ and, therefore, the closed geodesics intersect the hyperplane $X_{i}=0$ at 6 (generally complex) points. Since the geodesic is an elliptic curve in $\mathbb{C}^{3}$, it can be represented as an intersection of the quadric $Q$ with a cubic surface (30]).

The tangential 3:1 covering $\mathcal{G} \mapsto \mathcal{E}$ is associated to 3 -elliptic $\mathrm{KdV}$ solutions and dates back to works of Hermite and Halphen (24]). The genus 2 curve $\mathcal{G}$ is birationally equivalent to

$$
H=\left\{w^{2}=-\frac{1}{4}\left(4 z^{3}-9 g_{2} z-27 g_{3}\right)\left(z^{2}-3 g_{2}\right)\right\}
$$

which covers the elliptic curve with moduli $g_{2}, g_{3}$,

$$
\mathcal{E}_{1}=\left\{W^{2}=4 Z^{3}-g_{2} Z-g_{3}\right\} \subset(Z, W) .
$$


The covering is given by the relations

$$
Z=-\frac{1}{9} \frac{z^{3}-27 g_{3}}{z^{2}-3 g_{2}}, \quad W=\frac{2}{27} \frac{w\left(z^{3}-9 g_{2} z+54 g_{3}\right)}{\left(z^{2}-3 g_{2}\right)^{2}}
$$

and possesses 2 branch points. The last (second) holomorphic differential on $H$ reduces to an elliptic one:

$$
\frac{z d z}{w}=-\frac{2}{3} \frac{d Z}{W}
$$

In this case there is another $3: 1$ covering $\Pi_{2}: H \mapsto \mathcal{E}_{2}$ of the elliptic curve

$$
\begin{gathered}
\mathcal{E}_{2}=\left\{\mathcal{W}^{2}=\mathcal{Z}^{3}-G_{2} \mathcal{Z}-G_{3}\right\}, \\
G_{2}=\frac{27}{16}\left(g_{2}^{3}+9 g_{3}^{2}\right), \quad G_{3}=\frac{243}{32}\left(3 g_{3}^{2}-g_{2}^{3}\right),
\end{gathered}
$$

such that

$$
\mathcal{Z}=-\frac{1}{4}\left(4 z^{3}-9 g_{2} z-9 g_{3}\right), \quad \mathcal{W}=-w\left(z^{2}-\frac{3}{4} g_{2}\right), \quad \text { and } \quad \frac{d z}{w}=\frac{1}{3} \frac{d \mathcal{Z}}{\mathcal{W}}
$$

Let $\left(u_{1}, u_{2}\right)$ be local coordinates on $\operatorname{Jac}(H)$ associated to the holomorphic differentials $\left(k_{1} z d z / w, k_{2} d z / w\right), k_{1}, k_{2}=$ const. According to property (4) of the elliptic KdV solutions, for suitable normalization constants $k_{1}, k_{2}$ the period matrix of $\operatorname{Jac}(H)$ takes the form

$$
\left(\begin{array}{cccc}
2 \pi \jmath & 0 & \tau / 3 & 2 \pi \jmath / 3 \\
0 & 2 \pi \jmath & 2 \pi \jmath / 3 & \mathfrak{b} / 3
\end{array}\right)
$$

where $(2 \pi \jmath, \tau)$ and $(2 \pi \jmath, \mathfrak{b})$ are the normalized period matrices of $\mathcal{E}_{1}$ and $\mathcal{E}_{2}$ respectively.

Algebraic conditions for the closed geodesics. Under the birational equivalence of the curves $H$ and $\Gamma$, the structure of $H$ imposes conditions on the parameters $\left\{a_{1}, a_{2}, a_{3}, c_{1}\right\}=\left\{e_{1}, \ldots, e_{4}\right\}$ (squares of semi-axes and the constant of motion), for which the geodesics are closed, and which can be found explicitly. Notice that one can always set $e_{1}=a_{1}=1$ choosing appropriately $\alpha$ in (4.8). Then $\infty \in \Gamma$ is transformed either to one of roots $\pm \sqrt{3 g_{2}}$ or to one of the roots of $4 z^{3}-9 g_{2} z-g_{3}$ in (4.13). In the first case $e_{2}, e_{3}, e_{4}$ are positive, hence $Q$ is an ellipsoid, in the other case some of $e_{i}$ are negative, so $Q$ is a hyperboloid. In the latter case, reality condition implies that is the geodesic flow on the hyperboloid corresponds to unbounded motions, which we do not consider here.

These observations lead to the following sufficient conditions for a real geodesic on $Q$ to be closed.

Proposition 4.2 For given parameters $e_{1}=1, e_{2}>0$ the geodesic associated to the covering $H \rightarrow \mathcal{E}$ is closed if and only if one of the following pairs of conditions on $e_{3}$ and $e_{4}$ is satisfied

$$
\left(1+\frac{1}{e_{2}}+\frac{1}{e_{3}}\right)^{2}-4\left(\frac{1}{e_{2}}+\frac{1}{e_{3}}+\frac{1}{e_{2} e_{3}}\right)=0, \quad e_{4}=\frac{3 e_{2} e_{3}}{2\left(e_{2}+e_{3}+e_{2} e_{3}\right)}
$$


or

$$
9 e_{2}^{2} e_{3}^{2}-24 e_{2} e_{3}\left(e_{2}+e_{3}\right)+16 e_{2} e_{3}+16 e_{2}^{2}+e_{3}^{2}=0, \quad e_{4}=\frac{2 e_{2} e_{3}}{3 e_{2} e_{3}-2\left(e_{2}+e_{3}\right)} .
$$

If (4.18) is satisfied, then the following ordering holds

$$
\begin{gathered}
\text { for } \quad 0<e_{2}<\frac{1}{4}, \quad 0<e_{4}<e_{2}<e_{3}<1, \\
\text { for } \quad \frac{1}{4}<e_{2}<1 \quad 0<e_{4}<e_{2}<1<e_{3}, \\
\text { for } \quad 1<e_{2}<4, \quad 0<e_{4}<1<e_{2}<e_{3}, \\
\text { for } \quad e_{2}>4, \quad 0<e_{4}<1<e_{3}<e_{2} .
\end{gathered}
$$

Condition (4.19) is satisfied if and only if $e_{2}>1$, and the following ordering of roots holds:

$$
\begin{gathered}
\text { for } \quad 1<e_{2}<\frac{4}{3}, \quad 0<1<e_{2}<e_{3}<e_{4} \\
\text { for } \quad \frac{4}{3}<e_{2}<4, \quad 0<1<e_{3}<e_{2}<e_{4} \text {, } \\
\text { for } \quad e_{2}>4, \quad 0<1<e_{3}<e_{4}<e_{2} .
\end{gathered}
$$

Remark 4.2. The first condition corresponds to the case when $e_{1}=1$ is transformed into one of the roots of the polynomial $4 z^{3}-9 g_{2} z-g_{3}$, while in the second case 1 is transformed to $\sqrt{3 g_{2}}$.

The inverse relations are described by the following lemma.

Lemma 4.3 If $e_{2}, e_{3}, e_{4}$ is a solution of (4.18), then

$$
\beta=-\frac{1}{3}\left(1+\frac{1}{e_{2}}+\frac{1}{e_{3}}\right), \quad g_{2}=\frac{1}{3} \beta^{2}, \quad g_{3}=-\frac{4}{27}(\beta+1)\left(\beta+\frac{1}{e_{2}}\right)\left(\beta+\frac{1}{e_{3}}\right) .
$$

If condition (4.19) is satisfied, then

$$
\beta=-\frac{1}{2}, \quad g_{2}=\frac{1}{12}, \quad g_{3}=-\frac{1}{27}\left(\frac{1}{8}-\frac{1}{e_{2} e_{3} e_{4}}\right) .
$$

Proposition 4.2 and Lemma 4.3 are proved by pure calculations.

Remark 4.3. Since Proposition 4.2 imposes two independent conditions on the parameters $a_{2}, a_{3}, c$, not any triaxial ellipsoid has closed geodesics associated to the $3: 1$ tangential covering $H \rightarrow \mathcal{E}$. Naturally, this does not exclude existence of closed geodesics for the other types of hyperelliptic tangential coverings considered in [34, 35]. Description of such geodesics will be the subject of a future study. 


\section{$5 \quad$ Periodic billiard trajectories on a 2-dimensional quadric}

As shown in Section 2, a criterium for a generic billiard on an ellipsoid $Q$ to be periodic cannot be established in the complex setting. Below we restrict ourselves to 2-dimensional ellipsoid $Q$ for which the trajectories between impacts on $Q \cap Q_{d}$ are segments of the closed geodesics $X(s)$ described by Proposition 4.2. We study the following problem: given a family of such closed geodesics with an appropriate fixed caustic parameter $c$, for which boundary parameters $d$ the corresponding billiard orbit is periodic?

Correctness of the problem. As stated in the previous section, the closed geodesic $X(s)$ has 3 ingoing and 3 outgoing complex intersections with each component $D_{i, d}$ of $Q \cap Q_{d}$. Hence, to an initial set $(x, v), x \in D_{i, d}$ there correspond 3 possible branches $(\tilde{x}, \tilde{v})$, i.e., the restricted complex map $\mathcal{B}_{c}$ is 3 -valued. In this context the problem of its periodicity makes no sense, unless other properties are taken into account.

In the real domain there may exist one, two, or three real branches $\{(\tilde{x}, \tilde{v})\}$, and one naturally chooses a unique set $\left(\tilde{x}^{*}, \tilde{v}^{*}\right) \in\{(\tilde{x}, \tilde{v})\}$, such that $x$ and $\tilde{x}^{*}$ are subsequent points of intersection of $X(s)$ with $D_{i, d}$. However, $\left(\tilde{x}^{*}, \tilde{v}^{*}\right)$ cannot be distinguished form the other branches by purely algebraic methods. Yet the 3 branches have an important common property.

Lemma 5.1 Given an initial set $(x, v)$, the 3 sets $(\tilde{x}, \tilde{v})$ generate one and the same closed geodesic $\tilde{X}(s)$ on $Q$.

Indeed, according to Theorem 2.2 under the billiard map $\mathcal{B}_{c}$ the local coordinate $u_{2}$ on $\operatorname{Jac}(\Gamma)$ increases by the integral $\mathfrak{q}_{2}=\int_{E_{d-}}^{E_{d+}} \frac{\lambda d \lambda}{\mu}$, which depends on $d$ only. Hence, the images of the sets $(\tilde{x}, \tilde{v})$ in $\operatorname{Jac}(\Gamma)$ have the same coordinate $u_{2}$ and, therefore, they belong to the same trajectory of the $u_{1}$-flow on $\operatorname{Jac}(\Gamma)$.

It follows that the map $\mathcal{B}_{c}$ induces a map on the set of the closed geodesics, $\mathcal{F}_{c, d}: X(s) \mapsto \widetilde{X}(s)$, which is one-to-one.

If the orbit of $\mathcal{F}_{c, d}$ is periodic, then the orbit of the transcendental real one-toone map $\mathcal{B}_{c}^{*}:(x, v) \rightarrow\left(\tilde{x}^{*}, \tilde{v}^{*}\right)$ is periodic as well. Hence, an algebraic condition of periodicity for $\mathcal{F}_{c, d}$ gives us a correctly defined periodicity condition for the billiard on $Q$.

Periodicity conditions for $\mathcal{F}_{c, d}$. The map $\mathcal{F}_{c, d}$ can be described as the shift $u_{2} \mapsto u_{2}+\mathfrak{q}_{2}$ and under the birational transformation (4.8) the increment $\mathfrak{q}_{2}$ takes the form

$$
\mathfrak{q}_{2}=\int_{E_{\delta-}}^{E_{\delta+}} \frac{d z}{w}, \quad \delta=1 / d+\beta, \quad E_{\delta \pm}=(\delta, \pm \sqrt{R(\delta)}) \in H .
$$


Next, under the second covering $\Pi_{2}: H \mapsto \mathcal{E}_{2} \subset(\mathcal{Z}, \mathcal{W})$ described by (4.16), the above integral reduces to the elliptic one: $\mathfrak{q}_{2}=\frac{1}{3} \mathcal{I}_{\rho}$,

$$
\mathcal{I}_{\rho}=\int_{\Pi_{2}\left(E_{\delta-}\right)}^{\Pi_{2}\left(E_{\delta+}\right)} \frac{d \mathcal{Z}}{\sqrt{\mathcal{Z}^{3}-G_{2} \mathcal{Z}-G_{3}}}, \quad \Pi_{2}\left(E_{\delta \pm}\right)=\left(\rho, \pm \sqrt{\rho^{3}-G_{2} \rho-G_{3}}\right)
$$

where

$$
\rho=-\left(\delta^{3}-9 / 4 g_{2} \delta-9 / 4 g_{3}\right) .
$$

As follows from the structure of the period matrix of $\mathcal{G}$ in (4.17), the shift $u_{2} \mapsto u_{2}+\mathfrak{q}_{2}$ results in the same $u_{1}$-winding on $\operatorname{Jac}(\Gamma)$ if $\mathfrak{q}_{2}$ belongs to the lattice $\Lambda_{0}=\left\{2 \pi j \mathbb{Z}+\frac{1}{3} \mathfrak{b} \mathbb{Z}\right\}$. Hence, the orbit of $\mathcal{F}_{c, d}$ is periodic if and only if $\mathfrak{q}_{2}$ is a finite order point of $\Lambda_{0}$.

Since the period lattice $\Lambda=\{2 \pi \jmath \mathbb{Z}+\mathfrak{b} \mathbb{Z}\}$ of $\operatorname{Jac}\left(\mathcal{E}_{2}\right)$ is a sublattice of $\Lambda_{0}$, we conclude that $\mathcal{F}_{c, d}$ is periodic if, in particular, for an integer $m$, the integral $m \mathcal{I}_{\rho}$ is neutral in $\operatorname{Jac}\left(\mathcal{E}_{2}\right)$. (Notice that one should exclude trivial cases, when $\mathcal{I}_{\rho}$ is neutral in the lattice $\Lambda_{0}$.)

Then, by applying the Cayley-type condition (Theorem 3.1), one obtains an algebraic equation on the parameter $\rho$.

In practice, for large $m$, such equations appear to be rather tedious. We consider only the simplest nontrivial case $g=1, m=3$, when the condition (3.1) for the elliptic curve $\mathcal{E}_{2}$ takes the form

$$
\left|\begin{array}{ll}
S_{4} & S_{3} \\
S_{5} & S_{4}
\end{array}\right|=0
$$

$S_{j}$ being the coefficients of the expansion

$$
\begin{aligned}
\sqrt{\mathcal{Z}^{3}-G_{2} \mathcal{Z}-G_{3}} & \equiv \sqrt{(\mathcal{Z}-\rho)^{3}+P_{2}(\mathcal{Z}-\rho)^{2}+P_{1}(\mathcal{Z}-\rho)+P_{0}} \\
& =S_{0}+S_{1}(\mathcal{Z}-\rho)+S_{2}(\mathcal{Z}-\rho)^{2}+\cdots
\end{aligned}
$$

where

$$
P_{0}=\rho^{3}-G_{2} \rho-G_{3}, \quad P_{1}=3 \rho^{2}-G_{2}, \quad P_{2}=3 \rho .
$$

Condition (5.2) yields the equation

$$
\begin{aligned}
& \left(4 P_{0} P_{2}-P_{1}^{2}\right) \\
& \cdot\left(512 P_{0}^{4}+3 P_{1}^{6}-384 P_{0}^{3} P_{1} P_{2}-20 P_{0} P_{1}^{4} P_{2}+96 P_{0}^{2} P_{1}^{3}+64 P_{0}^{3} P_{2}^{3}+16 P_{0}^{2} P_{1}^{2} P_{2}^{2}\right)=0 .
\end{aligned}
$$

Substituting here expressions (5.3), we conclude that the integral $m \mathcal{I}$ is neutral in $\operatorname{Jac}\left(\mathcal{E}_{2}\right)$ if $\rho$ is a root of one of the following two equations

$$
\begin{aligned}
& 3 \rho^{4}-6 G_{2} \rho^{2}-12 G_{3} \rho-G_{2}^{2}=0 \\
- & \rho^{12}+22 G_{2} \rho^{10}+220 G_{3} \rho^{9}+165 G_{2}^{2} \rho^{8}+528 G_{2} G_{3} \rho^{7} \\
+ & \left(1776 G_{3}^{2}-92 G_{2}^{3}\right) \rho^{6}+264 G_{2}^{2} G_{3} \rho^{5}+\left(185 G_{2}^{4}-960 G_{2} G_{3}^{2}\right) \rho^{4} \\
+ & \left(80 G_{2}^{3} G_{3}-320 G_{3}^{3}\right) \rho^{3}+\left(624 G_{2}^{2} G_{3}^{2}-90 G_{2}^{5}\right) \rho^{2}+\left(896 G_{2} G_{3}^{3}-132 G_{2}^{4} G_{3}\right) \rho \\
+ & 512 G_{3}^{4}+3 G_{2}^{6}-96 G_{2}^{3} G_{3}^{2}=0 .
\end{aligned}
$$


Remark 5.1. The fact that equation (5.2) has 16 roots $\left\{\rho_{j}\right\}$ is natural: they correspond to 32 points $\left\{E_{\rho_{j} \pm}=\left(\rho_{j}, \pm \sqrt{\rho_{j}^{3}-G_{2} \rho_{j}-G_{3}}\right)\right\}$ on the curve $\mathcal{E}_{2}$ that satisfy conditions

$$
E_{\rho_{j}+} \neq E_{\rho_{j}-}, \quad 6 \mathcal{A}\left(E_{\rho_{j} \pm}\right) \equiv 0, \quad \text { where } \quad \mathcal{A}(P)=\int_{\mathcal{O}}^{P} \frac{d \mathcal{Z}}{\mathcal{W}}
$$

$\mathcal{O}$ being a Weierstrass point on $\mathcal{E}_{2}$. The corresponding 32 points $\mathcal{A}\left(E_{\rho_{j} \pm}\right) \in \operatorname{Jac}\left(\mathcal{E}_{2}\right)$ form a complete set of sixth order points $\{(\mathbb{Z} / 6 \mathbb{Z}) 2 \pi \jmath+(\mathbb{Z} / 6 \mathbb{Z}) \mathfrak{b} / 2\}$ of which the four second order points $\{0, \pi \jmath, \mathfrak{b} / 2, \pi \jmath+\mathfrak{b} / 2\}$ are excluded.

The resulting algorithm of constructing real periodic billiard trajectories on $Q$ consists of 4 steps.

1) Given a family of closed geodesics on $Q$ defined by parameters $e_{1}=1, e_{2}, e_{3}, e_{4}$ in Proposition 3.2, one finds the constant $\beta$ in (4.8) and moduli $g_{2}, g_{3}$ of the first elliptic curve $\mathcal{E}_{1}$ by using Lemma 4.3 and then, in view of (4.15), the parameters $G_{2}, G_{3}$ of the curve $\mathcal{E}_{2}$.

2) One finds the set $\left\{\rho_{j}\right\}$ of all roots of equations (5.4), (5.5) and then rejects the "trivial" ones, for which the integral $\mathcal{I}_{\rho}$ is neutral in the lattice $\Lambda_{0}$.

3) One computes the sets of corresponding admissible parameters $\delta$ and $d$ by solving the cubic equation (5.1) and the linear equation $\delta=1 / d+\beta$.

4) One selects the subset of real positive admissible values of $d$ for which the quadric $Q_{d} \subset \mathbb{R}^{3}$ has a nonempty intersection with the closed geodesics on $Q$, that is, the values that belong to segments $\left\{-\lambda\left(\lambda-e_{1}\right) \cdots\left(\lambda-e_{4}\right)>0\right\}$.

The parameters $e_{1}=1, e_{2}, e_{3}, e_{4}$ and the selected real values of $d$ characterize the billiard boundary $Q \cap Q_{d}$ and the caustics for which the nontrivial billiard orbits are periodic.

\section{Conclusion}

In this paper we studied algebraic geometrical properties of the billiard on a quadric $Q$ with bounces along its intersection with the confocal quadric $Q_{d}$.

If $Q$ is a 2-dimensional ellipsoid, we explicitly described a special class of closed geodesics associated to the 3 -soliton solutions of the $\mathrm{KdV}$ equation corresponding to the 3 -fold tangential covering $\mathcal{G} \mapsto \mathcal{E}_{1}$.

The existence of the second elliptic curve $\mathcal{E}_{2} \subset \operatorname{Jac}(\mathcal{G})$ in this case enabled us to apply the Cayley-type periodicity condition (Theorem [3.1) as an algebraic description of finite order points on $\mathcal{E}_{2}$ and, thereby, to obtain an explicit description of 3-periodic billiard orbits on $Q \cap Q_{d}$ whose points are joined by arches of the closed geodesics. As mentioned above, for period $m>3$ the periodicity conditions lead to quite tedious algebraic equations for the boundary parameter.

This approach cannot be directly extended to construct similar-type periodic billiard orbits on an $n$-dimensional quadric $Q$, since, instead of the complimentary 
elliptic curve $\mathcal{E}_{2}$, the Jacobian of $\mathcal{G}$ contains an $(n-1)$-dimensional Prym subvariety $\mathcal{A}_{n-1}$, which, in general, is not a Jacobian variety or its covering (except $n=3$ ).

Thus, we arrive at the problem of an algebraic description of finite-order points in $\mathcal{A}_{n-1}$ in terms of points of the curve $\mathcal{G}$, which deserves a separate studying.

\section{Acknowledgments}

We thank E.Previato, V. Dragović, and A.Treibich for useful discussions, as well as A. Perelomov for valuable remarks during preparation of the manuscript and indicating us the reference [8].

The second author (Yu.F.) acknowledges the support of grant BFM 2003-09504C02-02 of Spanish Ministry of Science and Technology.

This research was partially supported by GNFM-INdAM project "Onde nonlineari, struttura tau e geometria delle variet invarianti: il caso della gerarchia di Camassa-Holm".

\section{References}

[1] Abenda, S., Fedorov, Yu. On the weak Kowalevski-Painlevé property for hyperelliptically separable systems. Acta Appl. Math., 60 (2), (2000) 137-178

[2] Alber M., Fedorov, Yu. Algebraic geometrical solutions for certain evolution equations and Hamiltonian flows on nonlinear subvarieties of generalized Jacobians. Inverse Problems 17 (2001), no. 4, 1017-1042

[3] Audin M. Courbes algébriques et systèmes intégrables: géodésiques des quadriques. Expo. Math. 12, no.3 (1994), 193-226

[4] H. Airault, H. P. McKean, and J. Moser, Rational and elliptic solutions of the KdV equation and a related many-body problem. Commun. Pure Appl. Math. 30 , (1977), 94-148

[5] Birkhoff, J. D. Dynamical Systems. 4th edition. Providence, RI. 1966

[6] Belokolos E.D., Enol'ski V.Z. Reductions of theta-functions and elliptic finitegap potentials. Acta Appl. Math. 36, (1994), 87-117

[7] Belokolos E.D., Bobenko A.I., Enol'ski V.Z., Its A.R., and Matveev V.B. Algebro-Geometric Approach to Nonlinear Integrable Equations. Springer Series in Nonlinear Dynamics. Springer-Verlag 1994.

[8] von Braunmühl A. Notiz über geodätische Linien auf den dreiaxigen Flächen zweiten Grades welche durch elliptische Functionen dargestellen lassen. Math. Ann., 26 (1886), 151-153

[9] Cayley, A. Phil. Mag. 8 (1854), 339-348

[10] Calogero F. Exactly solvable one-dimensional many-body problems. Lett. Nuovo Cimento 13 (1975), 411-415 
[11] A.Clebsch, P.Gordan. Theorie der abelschen Funktionen. Teubner, Leipzig, 1866

[12] Chang, S.-J., Friedberg R. Elliptical billiards and Poncelets theorem. J. Math. Phys. 29, (1988) 1537-1550

[13] Chang, S.-J., Shi, K.-J. Billiard systems on quadric surfaces and the Poncelet theorem. J. Math. Phys. 30 (1989), no. 4, 798-804

[14] Chang, S.-J., Shi, K.-J. Elliptical billiard systems and the full Poncelet's theorem in $n$ dimensions. J. Math. Phys., 34 (1993), 2242-2256

[15] Dragović, V., Radnović, M. On periodical trajectories of the billiard systems within an ellipsoid in $\mathbb{R}^{n}$ and generalized Cayley's condition. J. Math. Phys. 39, No. 11, (1998), 5866-5869

[16] Dragović, V., Radnović, M. Cayley-type conditions for billiards within $k$ quadrics in $\mathbb{R}^{d}$. J. Phys. A: Math. Gen. 37 (2004), 1269-1276

[17] Dubrovin, B. A.; Novikov, S. P. A periodic problem for the Korteweg-de Vries and Sturm-Liouville equations. Their connection with algebraic geometry. (Russian) Dokl. Akad. Nauk SSSR, 219 (1974), 531—534.

[18] Enol'ski V., Richter P., Pronin A. Double Pendulum and $\theta$-Divisor J. Nonl. Sci. 13, (2003) 157-174

[19] Fedorov, Yu. Classical integrable systems related to generalized Jacobians. Acta Appl. Math. 55, 3, (1999), 151-201

[20] Gavrilov L., Perelomov A. On the explicit solutions of the elliptic Calogero system. J. Math. Phys. 40, no.12, (1999), 6339-6352

[21] Grammaticos, B., Ramani, A., Papageorgiu, V. Do integrable mappings have the Painlevé property? Phys. Rev. Lett. 67 (1991), 1825-1928

[22] Griffiths P., Harris J. A Poncelet theorem in space. Comment. Math. Helvetici. 52 (1977), 145-160

[23] Griffiths P., Harris J. On Cayley's explicit solution to Poncelets porism. LEnseignement Math. 24 (1978), 31-40

[24] Hermite C. Oeuvres de Charles Hermite. Vol. III, Gauthier-Villars, Paris, 1912.

[25] Knörrer H. Geodesics on the ellipsoid. Invent. Math. 59 (1980), 119-143

[26] Krichever I. Rational solutions of the Kadomtsev-Petviashvili equation and integrable systems on $n$ particles on the line. Funct. Anal. Appl. 12 (1977) 66-68

[27] Krazer A. Lehrbuch der ThetaFuntionen. Teubner, Leipzig, 1897 
[28] Markuschevich A. Introduction to the Classical Theory of Abelian Functions. Amer. Math. Soc. Transl. Ser.2, 96, Providence, USA, 1992

[29] Moser J. Various Aspects of Integrable Hamiltonian Systems. In: C.I.M.E. Lectures, Bressansone, Italy, 1978

[30] Perelomov A. Private communication.

[31] Previato, E. Poncelet theorem in space. Proc. Amer. Math. Soc. 127 (1999), no. $9,2547-2556$

[32] A. O. Smirnov. Elliptic solutions of the KdV equation. Math. Z. 45, (1989), 66-73

[33] A. O. Smirnov. Finite-gap elliptic solutions of the KdV equation. Acta Appl. Math. 36, (1994), 125-166

[34] Treibich A. and Verdier J. L. Tangential covers and sums of 4 triangular numbers. C. R. Acad. Sci. Paris 311, (1990), 51-54

[35] Treibich A. Hyperelliptic tangential covers, and finite-gap potentials. (Russian) Uspekhi Mat. Nauk 56 (2001), no. 6(342), 89-136; translation in Russian Math. Surveys 56 (2001), no. 6, 1107-1151

[36] Veselov, A.P. Integrable systems with discrete time, and difference operators. Funktsional. Anal. i Prilozhen. 22, (1988), no. 2, 1-13, English translation in Funct. Anal. Appl. 22, (1988), no. 2, 89-93

[37] Veselov, A.P. Complex geometry of the billiard on the ellipsoid and quasicrystallic curves. A seminar on Dynamical Systems. St. Petersburg, 1991

[38] Vanhaecke P. Integrable systems and symmetric product of curves. Math. Z., 227 (1998), no. 1, 93-127

[39] Weierstrass K. Über die geodätischen Linien auf dem dreiachsigen Ellipsoid. In: Mathematische Werke I, 257-266 